\title{
Índice Gilberto-Arruda: Avaliação do Impacto Social-acadêmico de Pesquisadores *
}

\author{
Lucas Henrique C. de Lima ${ }^{1}$, Gilderlanio S. Araújo ${ }^{2}$, \\ Pedro O. S. Vaz de Melo ${ }^{1}$, Ana Paula Couto da Silva ${ }^{1}$, Fabrício Benevenuto ${ }^{1}$ \\ ${ }^{1}$ Departamento de Ciência da Computação - Universidade Federal de Minas Gerais \\ ${ }^{2}$ Instituto de Ciências Biológicas - Universidade Federal de Minas Gerais \\ \{lhenriquecl, olmo, ana.coutosilva, fabricio\}@dcc.ufmg.br \\ gilderlaniosa@ufmg.br
}

\begin{abstract}
Studies show that the popularity of a user on Twitter is closely linked to the amount of followers that it has, while a large number of quotes in scientific environment is synonymous of high academic impact. Thus, this study has created a database of researchers with associated accounts on Twitter and Google Scholar in order to calculate the Gilberto-Arruda index. Results show that there are researchers associated with João Gilberto and others with Geisy Arruda, which means that some researchers have few followers but many quotes while others have many followers and few quotes. A web tool was developed and it allows researchers to see what is their Gilberto-Arruda index.
\end{abstract}

Resumo. Estudos apontam que a popularidade de um usuário no Twitter está muito vinculada à quantidade de seguidores que o mesmo possui, enquanto um grande número de citações no meio científico é sinônimo de alto impacto acadêmico. Neste contexto, este estudo criou uma base de dados de pesquisadores com contas no Twitter e no Google Acadêmico com o objetivo de calcular o indice Gilberto-Arruda. Os resultados encontrados mostram que existem pesquisadores que neste estudo são associados à João Gilberto e outros à Geisy Arruda, isto é, alguns pesquisadores possuem poucos seguidores porém muitas citações enquanto outros possuem muitos seguidores e poucas citações. Foi desenvolvida uma aplicação web que permite aos pesquisadores consultar qual o seu índice Gilberto-Arruda.

\section{Introdução}

As mídias sociais vêm transformando o modo que vivemos e trabalhamos [Mangold and Faulds 2009, Kaplan and Haenlein 2010, Safko 2010, Qualman 2010, Hanna et al. 2011]. Empresas, artistas e homens de negócio em geral usam as mídias sociais para divulgar os seus trabalhos e produtos, ampliar as suas redes de contatos e, fundamentalmente, expandir os seus negócios e aumentar os seus lucros. No entanto, a análise da participação de cientistas nas diversas mídias sociais e o consequente impacto dessas em suas carreiras é algo que ainda não foi completamente explorado na literatura [McClain and Neeley 2014]. Sabe-se, por exemplo, que redes sociais podem contribuir no meio acadêmico tanto para a troca de conhecimento quanto para formação de novas

*Esta pesquisa é financiada pelo CNPq, CAPES e FAPEMIG. 
colaborações entre pesquisadores [Van Noorden 2014]. Além disso, as mídias sociais também fornecem aos mesmos a oportunidade de divulgação de artigos, livros e o compartilhamento de pensamentos [Hadgu and Jáschke, Robert ].

Nesse contexto, o Twitter $^{1}$ é uma das principais redes sociais utilizadas por pesquisadores [Hadgu and Jáschke, Robert ]. De forma geral, o Twitter é um serviço social online de microblogging que permite a comunicação em tempo real entre os seus usuários através de pequenas mensagens de texto. Além de pesquisadores, seus usuários podem ser políticos, celebridades, artistas, ou pessoas comuns que simplesmente buscam uma plataforma para interagir com seus amigos e familiares. Assim, no meio científico existem pesquisadores que utilizam o microblogging para divulgar os seus trabalhos [McClain and Neeley 2014] e outros que os usam por motivos pessoais. Apesar das claras vantagens de se usar as mídias sociais em prol do crescimento profissional [Eysenbach 2011, Darling et al. 2013, Shuai et al. 2012], muitos pesquisadores ainda resistem e não as usam, alegando, por exemplo, falta de tempo ou questões relacionadas à privacidade [Focus 2009, Seaman and Tinti-Kane 2013].

Portanto, o presente trabalho oferece uma visão do engajamento de pesquisadores nas mídias sociais. Nosso objetivo é oferecer uma visão geral do engajamento de pesquisadores de diferentes áreas do conhecimento e correlacionar esse engajamento com a sua inserção no meio acadêmico, medida através do quão prolífico o pesquisador é. Mais especificamente, analisaremos se pesquisadores considerados influentes e populares no Twitter são também influentes e populares no Google Acadêmico ${ }^{2}$. Para isto, criamos uma base de dados de pesquisadores de diversas áreas do conhecimento que possuem perfis cadastrados tanto no Twitter quanto no Google Acadêmico. A criação desta base é um desafio por si só, pois é necessário criar métodos automáticos para fazer a associação de contas e a organização dos dados dos perfis públicos dos pesquisadores. A metodologia proposta expandiu de forma significativa a coleta de dados realizada por Hall [Hall 2014] e nos inspirou a criar um índice de impacto social-acadêmico, que correlaciona o impacto dos artigos de um pesquisador com a sua popularidade no Twitter.

Os resultados das análises mostram que existem pesquisadores que possuem um grande impacto acadêmico, mas que não são populares no Twitter. Por outro lado, existem aqueles que, relativamente, possuem baixo impacto acadêmico, porém são muito expressivos no meio social. Para medir tal variação, propomos o índice Gilberto-Arruda, que faz alusão a dois ícones brasileiros: João Gilberto e Geisy Arruda. João Gilberto é um cantor, violonista e compositor brasileiro, que apesar de ser considerado um dos pioneiros e mais importantes músicos da bossa nova, evita entrevistas e qualquer tipo de publicidade, sendo totalmente ausente nas mídias sociais. Por outro lado, Geisy Arruda é uma atriz, apresentadora, empresária e modelo brasileira, que apesar de não atuar na ciência e política, é presença constante nas mídias sociais e portais de celebridades. O índice Gilberto-Arruda é interessante porque, como mostraremos, há pouca correlação entre a popularidade de um pesquisador no Twitter e o impacto do seu trabalho no meio acadêmico. É importante ressaltar que este é o primeiro trabalho que analisa tal correlação em larga escala.

Este artigo encontra-se organizado da seguinte forma: na seção 2 apresentamos os trabalhos relacionados com o tema deste artigo. Na seção 3 apresentamos a metodologia

\footnotetext{
${ }^{1}$ https: //twitter.com/

${ }^{2}$ scholar.google.com
} 
de organização dos dados de pesquisadores. Na seção 4 apresentamos a análise dos dados coletados e na seção 5 apresentamos a ferramenta desenvolvida. Por fim, apresentamos as conclusões e trabalhos futuros na seção 6 .

\section{Trabalhos Relacionados}

A maneira como pesquisadores se comportam no Twitter vem sendo foco de alguns estudos atuais. O trabalho apresentado em [Hadgu and Jáschke, Robert ] desenvolveu uma metodologia para identificar pesquisadores no Twitter a partir de dados de conferências da ciência da computação. Os autores analisaram os dados a fim de entender como o meio de comunicação é utilizado pelos mesmos. Diferente de [Hadgu and Jáschke, Robert ], coletamos e caracterizamos não só pesquisadores da área de ciência da computação, mas acrescentamos pesquisadores de diferentes áreas, incluindo jornalismo, genômica, bioinformática, educação, entre outras.

O trabalho mais próximo ao nosso é o de Hall [Hall 2014], que propõe o índice Kardashian, definido como a razão entre o número de seguidores de um pesquisador no Twitter e o número de citações acadêmicas do mesmo. Nesse trabalho, a base de dados analisada limitou-se a apenas 40 cientistas selecionados aleatoriamente e que realizam pesquisas em genômica. Similar à Hall [Hall 2014], também propomos uma maneira de identificar pesquisadores populares nas redes sociais e não tão prolíficos no meio acadêmico. No entanto, ampliamos a base de 40 pesquisadores usada por Hall [Hall 2014] para milhares de pesquisadores de diferentes áreas, o que pode gerar conclusões mais amplas e estatisticamente validadas. Além disso, este estudo introduz a associação de pesquisadores à persona de João Gilberto, que oposto à Geisy Arruda, representa aqueles com alto impacto acadêmico porém com pouca popularidade nas redes sociais. Mais importante, nosso trabalho apresenta uma ferramenta que permite a expansão do número de pesquisadores na nossa base, não só cobrindo um maior número de pesquisadores e áreas, mas também permitindo sua expansão de forma ilimitada por mecanismos de crowdsourcing.

\section{Coleta de Dados}

A metodologia empregada para coleta de dados consiste em extrair atributos do Google Acadêmico e do Twitter para cada pesquisador. Seguimos três etapas principais: (i) coleta dos dados do Twitter partindo de uma lista inicial de pesquisadores; (ii) expansão da lista inicial para possíveis pesquisadores utilizando dados do Twitter; e (iii) associação das contas coletadas do Twitter ao Google Acadêmico, para a extração de índices de produção e das áreas de pesquisa relacionadas aos pesquisadores.

Na primeira etapa, a base inicial contém uma lista com 3.663 usuários do Twitter obtida pelo Cognos [Ghosh et al. 2012], que os classifica como possíveis pesquisadores influentes. Inicialmente, foi necessário coletar todos os dados referentes ao Twitter desses 3.663 identificadores, uma vez que o nome dos usuários é imprescindível para realizar consultas no Google Acadêmico. Além do nome, foram coletados também a localização, número de seguidores, número de listas, descrição, número de pessoas seguidas, número de pessoas marcadas como favoritas, perfil verificado (booleano), screen name e data de criação.

A partir desta lista inicial de usuários do Twitter, verificamos, através de uma API 
do Google Acadêmico ${ }^{3}$, a existência de contas de usuários comum aos dois sistemas. Para isso, foi feita uma consulta na API do Google Acadêmico usando o nome do usuário que o mesmo fornece na sua conta do Twitter. No total, 276 (7,5\%) usuários tiveram pelo menos um resultado retornado na busca no Google Acadêmico. Disponível em:cran.rproject.org/web/packages/scholar/.

A partir de então, como maneira de expandir a base de dados, coletamos no Twitter todos os seguidores desses 276 usuários que, possivelmente, possuem conta tanto no Twitter quanto no Google Acadêmico. Essa coleta retornou 566.368 usuários. Desses 566.368 usuários, 24.957 (4,4\%) possuem contas que têm o mesmo nome tanto no Google Acadêmico quanto no Twitter. Como um usuário no Twitter pode seguir mais de um dos possíveis pesquisadores identificados até o momento, nomes repetidos foram encontrados. Retiradas as duplicatas (4.493), a base passou a ter 20.464 pesquisadores.

Assim, a partir desta nova e filtrada base de dados, que contém 20.464 tuplas formadas por uma conta no Twitter a outra no Google Acadêmico, conectadas pelo nome que consta em ambas as contas, foi necessário realizar uma nova coleta de dados, agora no site da Wikipedia ${ }^{4}$. Foi observado que na lista gerada pela metodologia descrita acima, nomes como Albert Einstein, Karl Marx e Sigmund Freud estavam presentes, pessoas essas que já são falecidas. Dessa forma, para cada usuário, foi realizada uma requisição na página da Wikipedia por seus respectivos nomes e, caso a mesma retornasse data de falecimento, o usuário seria descartado da base final. Dos 20.464 potenciais pesquisadores, 210 (1\%) foram eliminados da base por serem pessoas falecidas. A base, então, passou a conter 20.254 pesquisadores.

Por fim, na terceira e última etapa do processo, realizamos uma coleta no Google Acadêmico dos dados de produção dos potenciais pesquisadores até então obtidos. Tais dados são: quantidade total de citações, $h$-index [Hirsch 2005], i10-index ${ }^{5}$, campos de estudo, instituição vinculada e webpage associada. Nesta etapa, observou-se que uma conta do Twitter, então associada à uma conta no Google Acadêmico com nome idêntico à primeira, poderia representar pessoas diferentes. Dessa maneira, foram selecionados apenas os pesquisadores que possuem, no texto de descrição do perfil de usuário do Twitter, pelo menos um dos campos de estudo retornado pelo Google Acadêmico. Além disso, para assegurar que as contas no Twitter e no Google Acadêmico estão associadas à mesma pessoa, eliminamos da base aqueles usuários que tiveram mais de um nome idêntico e mais de um e-mail confirmado para uma requisição. Com a realização de todos esses filtros, a base de dados, que antes possuía 20.254 pesquisadores, passa a conter 1.898 pesquisadores e torna-se a base de pesquisadores final utilizada neste trabalho.

A fim de avaliar a qualidade da metodologia utilizada para a associação de contas do Google Acadêmico à contas do Twitter, analisamos manualmente uma amostra aleatória de 150 dos 1.898 pesquisadores até então coletados. Dessa amostra de 150 usuários, foi possível confirmar a equivalência entre as contas de 146 usuários (97,3\%), enquanto dois apresentaram associatividade inconclusiva e dois representaram pessoas

\footnotetext{
${ }^{3} \mathrm{O}$ scholar é um pacote de funções, implementado em linguagem R, para realizar consultas ao Google Acadêmico. O scholar provê funções de predição do fator de impacto além de possibilitar a extração de dados dos perfis de pesquisadores, como o número de citações e fatores de impacto.

${ }^{4}$ https: / / www.wikipedia.org/

${ }^{5}$ http://googlescholar.blogspot.com.br/2011/11/google-scholar-citations-open-to-all.html
} 
distintas. Com isso, dado que o erro de associação esperado é baixo, utilizamos a mesma metodologia para a realização de análises quanto às métricas de citação e campos de estudo, além da construção de uma aplicação web que irá confrontar o impacto acadêmico gerado no ambiente social. É importante ressaltar que o método de associação de contas proposto por [Hadgu and Jáschke, Robert ] tem uma taxa de acerto esperada de apenas $73 \%$.

\section{Análise e Discussão}

Nesta seção iremos caracterizar o perfil e a influência dos 1.898 pesquisadores da nossa base de dados. Primeiramente, traçamos o perfil dos pesquisadores analisando a distribuição geográfica e seus tópicos de pesquisa (Seção 4.1). A seguir, na Seção 4.2, analisamos o impacto acadêmico do pesquisador, através do $h$-index, i10-index e total de citações, e a influência social, através do número total de seguidores no Twitter. A Seção 4.3 apresenta o índice Gilberto-Arruda, que mede a relação entre a produção/impacto acadêmico e popularidade nas redes sociais para um dado pesquisador.

\subsection{Perfil dos Pesquisadores}

A Figura 1 apresenta a distribuição geográfica aproximada (por país) dos pesquisadores da base de dados, sendo que $46,46 \%$ estão no continente norte americano, 40,66\% no continente europeu, $0,89 \%$ no continente africano, $3.56 \%$ no continente asiático, $7,45 \%$ no continente oceania e $0.95 \%$ no continente sul americano. O coeficiente de Gini ${ }^{6}$ do número de pesquisadores por país ${ }^{7}$ é de 0,43 , o que indica uma leve desigualdade na distribuição de pesquisadores por país.

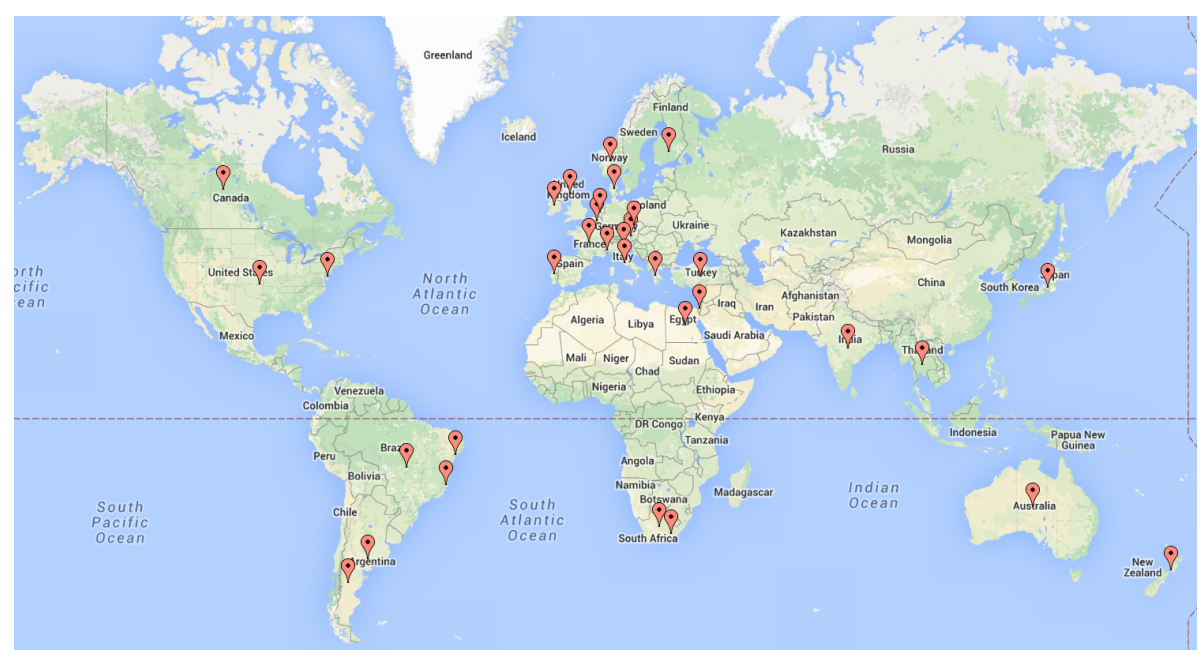

Figura 1. Geolocalização aproximada dos pesquisadores coletados.

Para verificar a interdisciplinaridade dos pesquisadores, foram analisados os 3.752 tópicos de pesquisa da base de dados coletada. Entre os termos analisados, os mais frequentes incluem machine learning, social media, bioinformatics, education e artificial

\footnotetext{
${ }^{6}$ O Coeficiente de Gini é uma medida de desigualdade, comumente utilizada para calcular a desigualdade de distribuição de renda, mas pode ser usada para qualquer distribuição. Ele consiste em um número entre 0 e 1 , onde 0 corresponde à completa igualdade(ex: todos têm a mesma renda) e 1 corresponde à completa desigualdade (ex: uma pessoa tem toda a renda).

${ }^{7}$ Considerando somente os países que possuem pesquisadores na nossa base.
} 
intelligence. A Figura 2(a) apresenta a distribuição da frequência dos termos de pesquisa que são relacionados a no mínimo 10 pesquisadores. A Figura 2(b) apresenta os 20 termos mais frequentes em nossa base. Note que grande parte deles está relacionado à linhas de pesquisa da Ciência da Computação. Isso sugere que é mais provável ter pesquisadores da Ciência da Computação com contas tanto no Twitter quanto no Google Acadêmico do que pesquisadores de outras áreas.

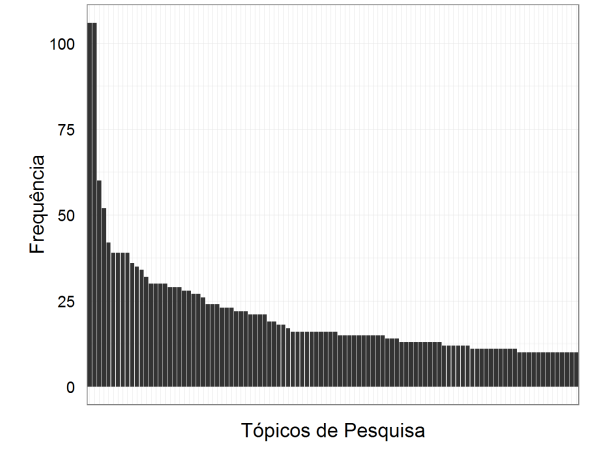

(a) Distribuição de frequência para os 104 termos relacionados a no mínimo 10 pesquisadores.

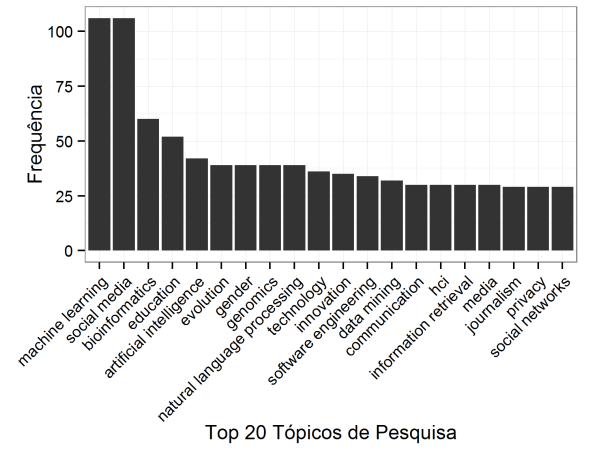

(b) Distribuição de frequência para os 20 termos mais frequentes.

Figura 2. Distribuição da frequência dos termos de pesquisa.

Outra coisa que observamos nos dados é que os pesquisadores analisados tendem a possuir um perfil interdisciplinar, dado que todos pesquisadores possuem 2 ou mais tópicos de pesquisa associados aos seus respectivos perfis no Google Acadêmico. Em média o número de tópicos de pesquisa por pesquisador é de 4,1 (com mediana de 4). A Figura 3 apresenta esta distribuição. Observe que a distribuição é bem desigual, em que o máximo de tópicos associados é de 5, no caso de 884 pesquisadores, 4 tópicos para 478 pesquisadores, 3 termos para 402 pesquisadores e 2 termos para 134 pesquisadores.

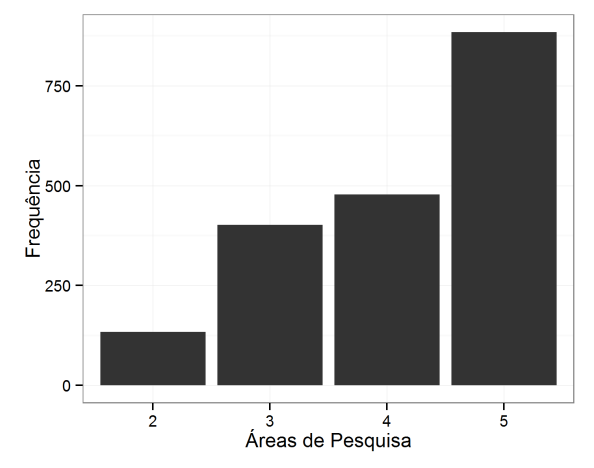

Figura 3. Frequência de termos de pesquisa associados por pesquisador.

\subsection{Participação dos Pesquisadores}

\section{Meio Acadêmico}

A partir da base de dados coletada, apresentamos a caracterização dos pesquisadores em relação aos índices de produção acadêmica coletados a partir do Google Acadêmico. A 
média de citações entre os pesquisadores coletados é de 1.112 citações e mediana de 160 citações, a partir da distribuição apresentada na Figura 4(a). Dado o desvio padrão da distribuição (3476.4), concluímos que os dados estão dispersos, devido a presença de valores atípicos (outliers), como é o caso dos pesquisadores que possuem mais do que 70 mil citações.

$\mathrm{O}$ h-index e il0-index apresentam distribuições diferentes (de acordo com o teste de Wilcoxon ${ }^{8}$, com $\left.\mathrm{p}<2.2 \mathrm{e}-16\right)$ e correlação de Pearson igual a $0.93(\mathrm{p}<2.2 \mathrm{e}-16)$. A média do $h$-index entre os pesquisadores é igual a 9.6 e mediana de 6 . Já a distribuição do i10-index apresenta média de 13.7 e mediana de 9. Para as distribuições dos índices (ver Figuras 4(b) e 4(c)), o desvio padrão correspondente é 11.07 e 22.92, respectivamente.

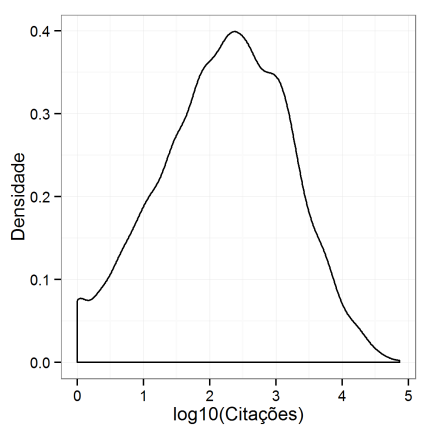

(a) Distribuição do número de citações.

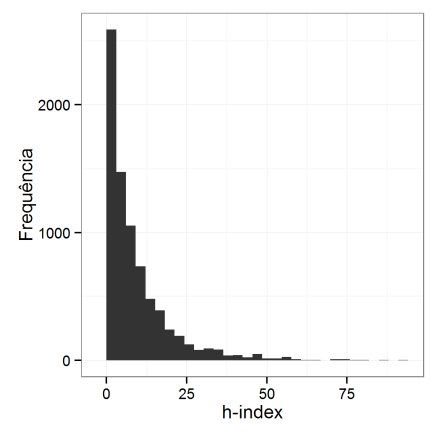

(b) Distribuição do h-index.

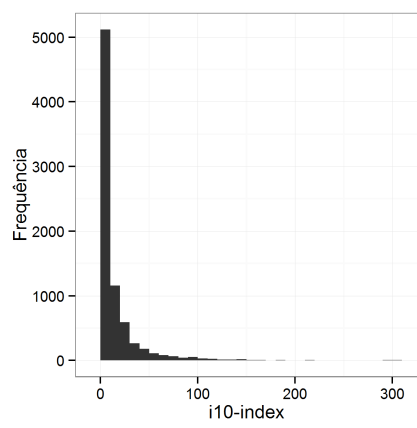

(c) Distribuição do i10-index.

Figura 4. Distribuição das citações, $h$-index e i10-index dos pesquisadores.

\section{Redes Sociais}

Neste trabalho, um pesquisador é considerado popular nas redes sociais se o mesmo possui muitos seguidores em seu Twitter. A Figura 5 apresenta a distribuição dos seguidores do Twitter dos pesquisadores coletados. Observe que muitos pesquisadores possuem poucos seguidores no Twitter, isto é, baixa popularidade. A média de seguidores é de 1.002, mediana igual 358,5, e desvio padrão correspondente de 3.532. A distribuição também é bastante desigual e se assemelha a uma distribuição lognormal (eixo horizontal está em escala logarítmica).

\subsection{Impacto social-acadêmico}

Caracterizadas as distribuições das participações dos pesquisadores no meio acadêmico e nas redes sociais, analisaremos agora a correlação entre ambas. Para isso, investigamos a relação entre as métricas de citação fornecidas pelo Google Acadêmico e o número de seguidores de um determinado pesquisador. A partir das Figuras 6, 7 e 8, é possível notar que existe baixa correlação entre o número de seguidores de um pesquisador e os índices de citação.

A Tabela 1 apresenta os coeficientes de correlação de Pearson e Spearman para as relações entre o número de seguidores do Twitter de um pesquisador e suas respectivas

\footnotetext{
${ }^{8} \mathrm{O}$ teste de Wilcoxon é um método não-paramétrico utilizado para comparar duas amostras pareadas.
} 


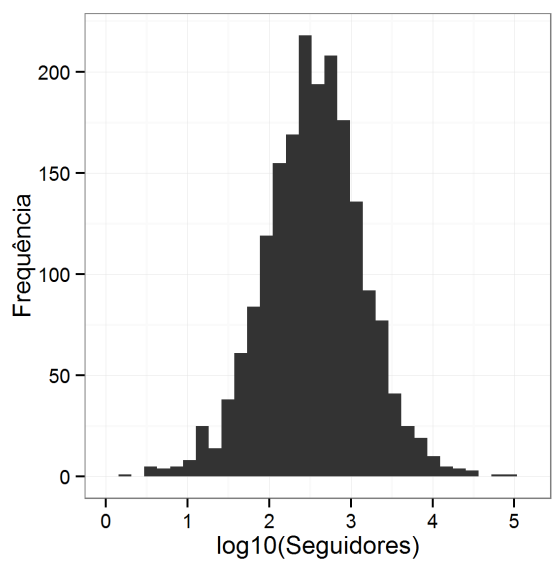

Figura 5. Distribuição do número de seguidores.

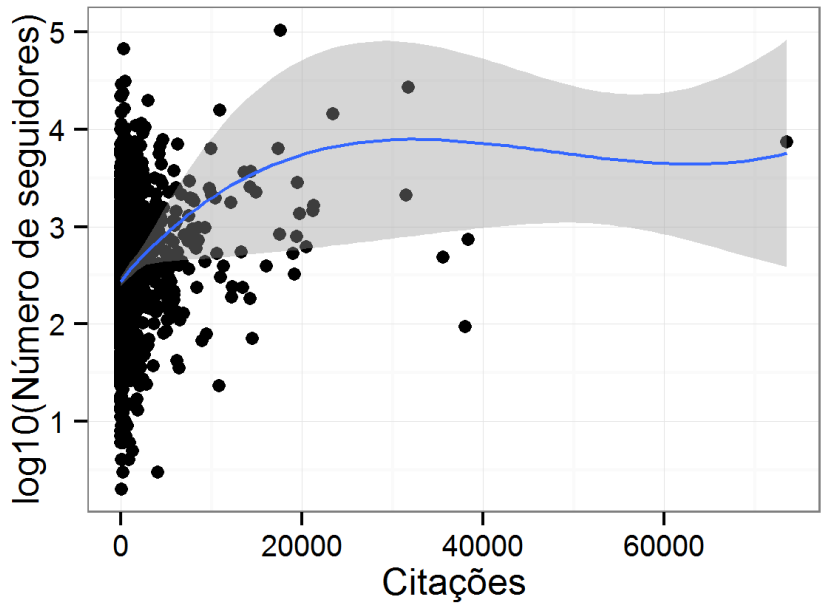

Figura 6. Número de Seguidores x Número de Citações.

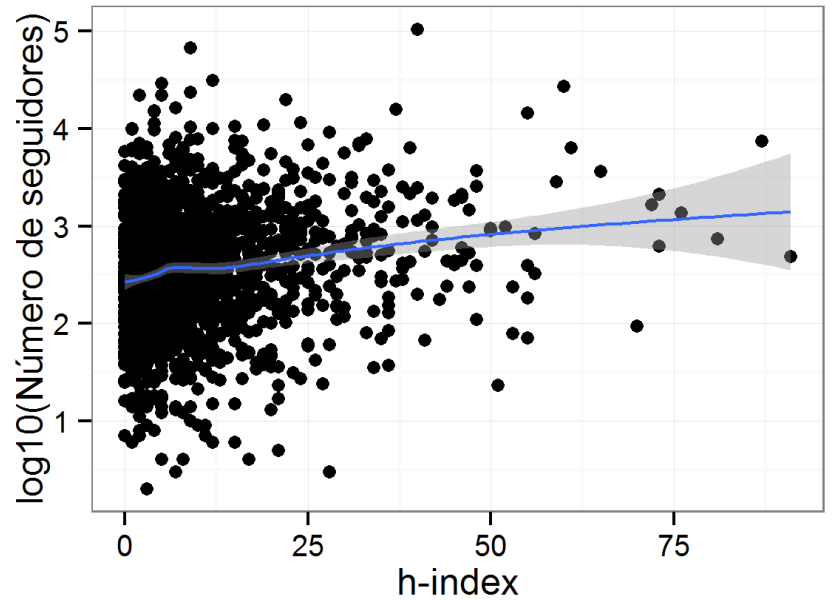

Figura 7. Número de Seguidores $\mathrm{x} h$-index. 


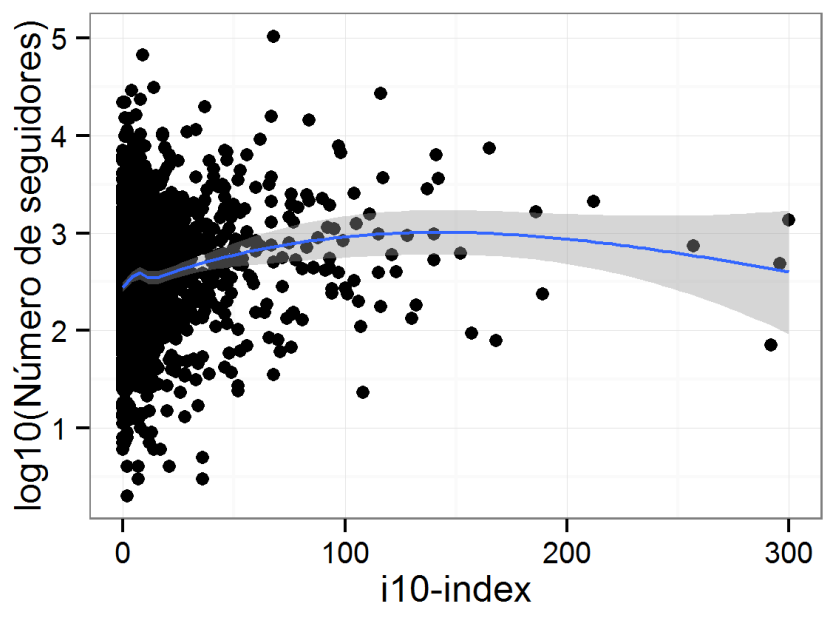

Figura 8. Número de Seguidores x i10-index.

métricas de citação ( $\mathrm{p}$-value $<3.17 \mathrm{e}-5$ para todos os casos). Os resultados apresentados indicam coeficientes próximos à 0 . Assim, isso sugere que as variáveis são independentes uma da outra, ou seja, a popularidade de um pesquisador no Twitter não influencia, necessariamente, a sua produção e impacto no meio acadêmico (e vice-versa).

\begin{tabular}{|c|c|c|}
\hline Relação & Coef. de Pearson & Coef. de Spearman \\
\hline Número de Seguidores/Número de Citações & cor 0.172 & rho 0.152 \\
\hline Número de Seguidores/i10-index & cor 0.094 & rho 0.152 \\
\hline Número de Seguidores/h-index & cor 0.122 & rho 0.158 \\
\hline
\end{tabular}

Tabela 1. Coeficiente de Pearson e Spearman

\section{4. Índice Gilberto-Arruda}

Considerando que as variáveis de impacto acadêmico e impacto social são independentes uma da outra, este trabalho criou o índice Gilberto-Arruda, que é baseado no índice Kardashian [Hall 2014]. Diferente do índice Kardashian, que é definido como a razão entre o número de seguidores e o número de citações de um pesquisador, o índice GilbertoArruda indica, em uma escala que varia de 0 a 1, a relação do índice Kardashian de um determinado pesquisador quando em comparação aos outros pesquisadores da base de dados. Mais especificamente, o índice Gilberto-Arruda de um pesquisador com índice Kardashian $x$ é a função distribuição acumulada empírica $F(x)=P(X \leq x)$ de $x$, em que $X$ é a variável aleatória que representa a distribuição dos índices Kardashian dos pesquisadores da nossa base. Assim, pesquisadores com índice Gilberto-Arruda maior do que 0.5 são mais associados à Geisy Arruda do que à João Gilberto. Caso o índice Gilberto-Arruda seja menor do que 0.5, os pesquisadores são mais associados à João Gilberto do que à Geisy Arruda.

A relação entre o índice Kardashian e o índice Gilberto-Arruda é apresentada na Figura 9. Valores do índice Gilberto-Arruda próximos à 1 e índice Kardashian negativo, na escala apresentada, denotam aqueles pesquisadores com baixo índice de produção acadêmica e maior popularidade nas redes sociais. Por outro lado, valores positivos do 
índice Kardashian e valores próximos à 0 para o índice Gilberto-Arruda denotam os pesquisadores com baixa popularidade nas redes sociais e alto índice de produção acadêmica. É interessante observar que há pesquisadores em ambos os extremos, mas a grande maioria deles está próximo ao centro, sendo que aproximadamente $60 \%(1142)$ dos pesquisadores possuem índice Kardashian entre 0.1 e 10.

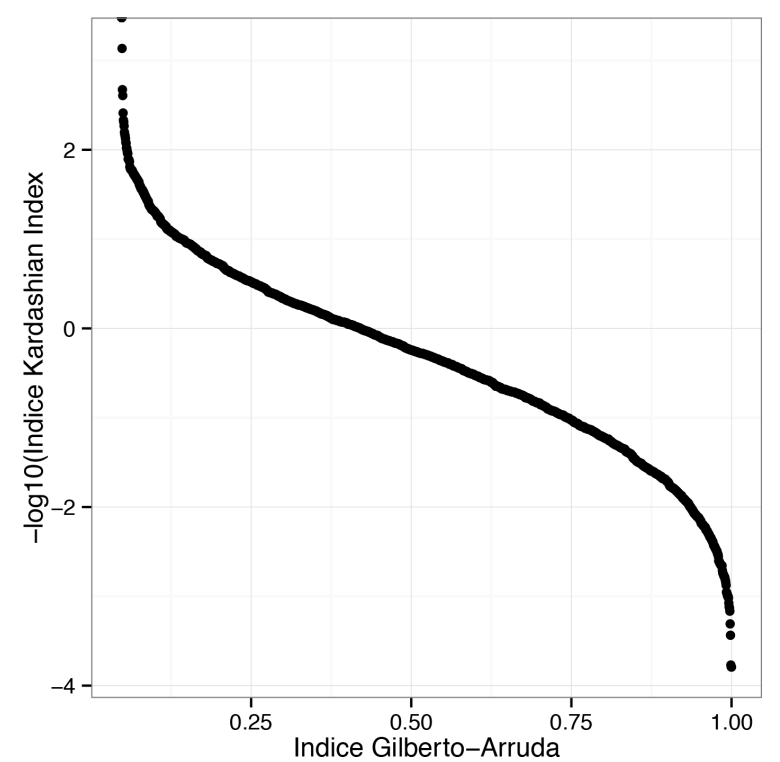

Figura 9. Relação do índice Kardashian e Índice Gilberto-Arruda (percentil)

\section{Aplicação Web}

Considerando o número de pesquisadores coletados neste trabalho com contas no Twitter e no Google Acadêmico, foi desenvolvida uma ferramenta que permite aos mesmos e a outros pesquisadores a consulta do seu índice Gilberto-Arruda, que compara o índice Kardashian dos pesquisadores da base com o índice Kardashian requerido. A página inicial da ferramenta é mostrada pela Figura 10.

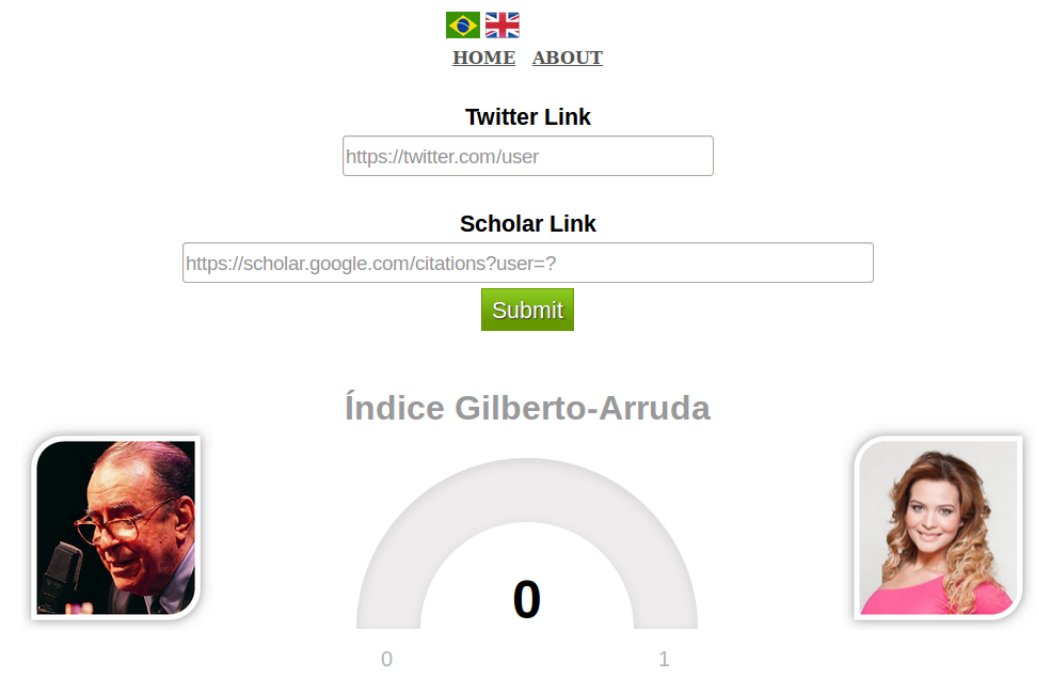

Figura 10. Página Inicial 
Para consultar o índice Gilberto-Arruda de um pesquisador, é necessário inserir o link referente à sua página no Twitter e no Google Acadêmico. O resultado próximo à 1 indica que o pesquisador possui um Índice Kardashian maior do que os outros pesquisadores e, por isso, está mais associado à Geisy Arruda do que à João Gilberto. O oposto acontece para um valor retornado próximo à zero.

Além de consulta, os pesquisadores que submeterem seus respectivos links nesta aplicação poderão ser inseridos na base de dados. Caso o nome da conta do Twitter seja similar ao nome da conta do Google Acadêmico fornecido, esse pesquisador terá seus dados inserido na base de pesquisadores. É importante a realização desse filtro para que a base não seja contaminada com contas de usuários que não representam a mesma pessoa. Dessa forma, além de permitir consulta, a ferramenta foi criada com o objetivo de possibilitar a expansão da base de pesquisadores e posteriormente possibilitar o cálculo do índice Gilberto-Arruda por áreas de conhecimento.

\section{Conclusões}

Neste trabalho, desenvolvemos uma metodologia capaz de associar e coletar dados de contas de pesquisadores do Twitter de diversas áreas do conhecimento à contas no Google Acadêmico. Constatamos que, na base de dados coletada, existe uma heterogeneidade do perfil dos pesquisadores principalmente quanto à localização geográfica e quanto aos campos de estudo dos mesmos.

Concluímos ainda que a grande parte dos pesquisadores possuem baixa popularidade social, uma vez que a maioria possui poucos seguidores no Twitter. Considerando então que as variáveis de impacto acadêmico e popularidade social são independentes, foi criado o índice Gilberto-Arruda, baseado no índice Kardashian, que indica em uma escala que varia de 0 a 1 a relação do índice Kardashian de um pesquisador requerido quando em comparação com os outros pesquisadores da base.

Finalmente, foi desenvolvida uma aplicação web que possibilita aos pesquisadores a consulta do seu respectivo índice Gilberto-Arruda e sua associação à João Gilberto ou Geisy Arruda. Com o fornecimento dos links das contas associadas ao Twitter e Google Acadêmico, além do resultado que é apresentado ao pesquisador, o mesmo pode ser inserido na base de pesquisadores já coletada e possibilitar expansão da base de dados.

\section{Trabalhos Futuros}

Para trabalhos futuros estamos interessados em verificar as relações existentes entre os pesquisadores coletados. Além disso, pretendemos avaliar os aspectos de colaboração acadêmica entre pesquisadores e a ligação dos mesmos através dos relacionamentos no Twitter, e analisar como pesquisadores de diferentes áreas do conhecimento conectam-se entre si.

Finalmente, pretendemos realizar um estudo acerca das métricas de citação e do número de seguidores de um determinado pesquisador além de verificar o comportamento de pesquisadores associados a João Gilberto e à Geisy Arruda quanto aos seus posts no Twitter, isto é, analisar se há a exposição de artigos, trabalhos e outros assuntos referentes ao mundo acadêmico. 


\section{Referências}

Darling, E., Shiffman, D., Cote, I., and Drew, J. (2013). The role of Twitter in the life cycle of a scientific publication. Ideas in Ecology and Evolution, 6:31.

Eysenbach, G. (2011). Can tweets predict citations? Metrics of social impact based on Twitter and correlation with traditional metrics of scientific impact. Journal of medical Internet research, 13(4).

Focus, F. (2009). Twitter in Higher Education: Usage Habits and Trends of Todayâs College Faculty. Madison, WI: Magna Publications.

Ghosh, S., Sharma, N., Benevenuto, F., Ganguly, N., and Gummadi, K. (2012). Cognos: crowdsourcing search for topic experts in microblogs. In Proceedings of the 35th international ACM SIGIR conference on Research and development in information retrieval, pages 575-590. ACM.

Hadgu, A. T. and Jáschke, Robert, booktitle=Proceedings of the 2014 ACM conference on Web science, p. y. o. Identifying and analyzing researchers on twitter.

Hall, N. (2014). The kardashian index: a measure of discrepant social media profile for scientists. Genome biology, 15(7):424.

Hanna, R., Rohm, A., and Crittenden, V. L. (2011). We're all connected: The power of the social media ecosystem. Business Horizons, 54(3):265-273.

Hirsch, J. E. (2005). An index to quantify an individual's scientific research output. Proceedings of the National academy of Sciences of the United States of America, 102(46):16569-16572.

Kaplan, A. M. and Haenlein, M. (2010). Users of the world, unite! The challenges and opportunities of Social Media. Business Horizons, 53(1):59-68.

Mangold, W. G. and Faulds, D. J. (2009). Social media: The new hybrid element of the promotion mix. Business Horizons, 52(4):357-365.

McClain, C. and Neeley, L. (2014). A critical evaluation of science outreach via social media: its role and impact on scientists. F1000Research, 3.

Qualman, E. (2010). Socialnomics: How Social Media Transforms the Way We Live and Do Business. Business professional collection. Wiley.

Safko, L. (2010). The Social Media Bible: Tactics, Tools, and Strategies for Business Success. Wiley.

Seaman, J. and Tinti-Kane, H. (2013). Social media for teaching and learning. Pearson Learning Solutions.

Shuai, X., Pepe, A., and Bollen, J. (2012). How the Scientific Community Reacts to Newly Submitted Preprints: Article Downloads, Twitter Mentions, and Citations. PLoS ONE, 7(11).

Van Noorden, R. (2014). Online collaboration: Scientists and the social network. Nature, 512(7513):126-129. 